\title{
PROTON MAGNETIC RESONANCE SPECTROSCOPY IN HUNTINGTON'S DISEASE ACCOMPANYING NEUROBORRELIOSIS
}

\author{
Helena Sarac $^{1,2,3}$, David Ozretic ${ }^{4}$, Neven Henigsberg ${ }^{2,3}$, Pero Hrabac ${ }^{2,3}$, \\ Ivan Bagaric $^{5}$ \& Lucija Bagaric-Krakan \\ ${ }^{1}$ Department of Neurology, University Hospital Zagreb, Zagreb, Croatia \\ ${ }^{2}$ Croatian Institute for Brain Research, Zagreb, Croatia \\ ${ }^{3}$ School of Medicine, University of Zagreb, Zagreb, Croatia \\ ${ }^{4}$ Department of Radiology, Univesrity Hospital Center Zagreb, Zagreb, Croatia \\ ${ }^{5}$ Medical School University of Mostar, Department of Public Health, Mostar, Bosnia and Herzegovina \\ ${ }^{6}$ Department of Psychiatry, University Hospital Center Zagreb, Zagreb, Croatia
}

received: 28.11.2016;

revised: 1.2.2017;

accepted: 28.3.2017

\section{INTRODUCTION}

Pathophysiology of HD is still unclear, and potential HD biomarkers are lacking. Discrepant findings have been reported in the literature about the role of the proton magnetic resonance spectroscopy (MRS) as an biomarker of the Huntington's disease (HD) and neuroborreliosis.

Recently, the autors of the largest longitudinal study reported putaminal MRS measurements of myo-inositol (mI) and N-acetylaspartate (NAA) as a potential biomarkers of HD onset and progression (Sturrock et al. 2010). Only one report demonstrated MRS abnormalities in patients with neuroborreliosis (Ustymovicz et al. 2004). Herein, we quantified metabolic abnormalities by using proton MRS and assesed the extent of structural abnormalities in brain MRI in three HD gene carriers; one patient with HD and neuroborreliosis, one adult-onset HD patient and one presymnptomatic HD (pre-HD) individual.

\section{CASE DESCRIPTIONS}

\section{Patient 1}

A 52-year-old woman presented to neurologic unit because of mood changes and unsteady gait for two months. She reported tick bites with annular rash on several occasions over the last year which subsided without antimicrobial therapy. Clinical examination revealed behavioral changes, right-sided hemidystonia and restlesness. Her deep tendon reflexes were brisk. Her Mini-Mental State Examination (MMSE) score was 17 of 30. Peripheral blood cell counts disclosed leukocytosis. Autoimmunity screening including anti-nuclear antibodies (ANA), anti-double stranded DNA (antidsDNA), anti-Smith (anti-Sm), antinuclear ribonucleoprotein (anti-nRNP), anti-glutamic acid decarboxylase (anti-GAD), c-neutrophil 2 cytoplasmic antibodies (ANCA) and myeloperoxidase antibodies (anti-TPO), were negative.
Lyme serology was performed; enzyme-linked immunosorbent assay, ELISA: IgM was positive and IgG was positive in the serum with a titer of 1:512 (cutoff 1:256). The cerebrospinal fluid (CSF) contained 55 lymphocytes and the total protein was $0.72 \mathrm{~g} / \mathrm{l}$. The titer of antibodies against Borrelia burgdorferi $(\mathrm{Bb})$ in CSF was 1:256 (cut-off 1:8).

Bacteriological cultures were negative, as serological studies for neurotropic viruses and tumour markers. Brain magnetic resonance imaging (MRI) showed multifocal punctiform signal hyperintensities (SH) over the white matter and marked cortico-subcortical atrophy (Figure 1A, B). Indeed, the ratios of $\mathrm{N}$-acetylaspartate (NAA) to creatine (Cr) (NAA/Cr), choline (Cho) to $\mathrm{Cr}$ (Cho/Cr), myo-inositol (mI) to $\mathrm{Cr}(\mathrm{mI} / \mathrm{Cr}$ ), $\mathrm{Lac} / \mathrm{Cr}$, were obtained by MRS (Figure 1C, D). Spectra acquired from the normal-appearing left putamen and white matter of the frontal lobe showed fourfold increased lactate (Lac) centered at $1.3 \mathrm{ppm}$ at $1.5 \mathrm{~T}$, increased glutamine-glutamate (Glx) at $2.3 \mathrm{ppm}$ and increased $\mathrm{Cho} / \mathrm{Cr}$ ratio at 3.2 $\mathrm{ppm}$. The $\mathrm{mI} / \mathrm{Cr}$ ratio was slightly decreased at $3.5 \mathrm{ppm}$ and NAA/Cr was normal at $2.0 \mathrm{ppm}$ (Table 1).

Owing to the suspected neuroborreliosis, the patient was treated with ceftriaxone $2 \mathrm{~g} / \mathrm{daily} / 4$ weeks. One week after initiation of antibiotic treatment, the anti-Bb titre was 1:256 in the serum and 1:128 in the CSF. Three months later, the patient reached full recovery of her neurologic symptoms. One year after initiation of antibiotic treatment, the patient developed abnormal involuntary movements. On neurological examination, she exhibited choreatic movements, shrugging of shoulders, swaying of the trunk and unsteadiness of gait. Speech was slurred and staccato in nature. There was impersistence of gaze, and she was unable to move her eyes in a rapid saccade. Her cognitive abilities were impaired and her mood fluctuate from anxiety to depression. Tests for anti-Bb were negative in the serum and CSF. Brain MRI (Figure 1E) revealed that white matter SH remained substantially unchanged, but we noted striatal atrophy producing an increase in intercaudal 3 distance and ventricular enlargement (,box-shape“), 

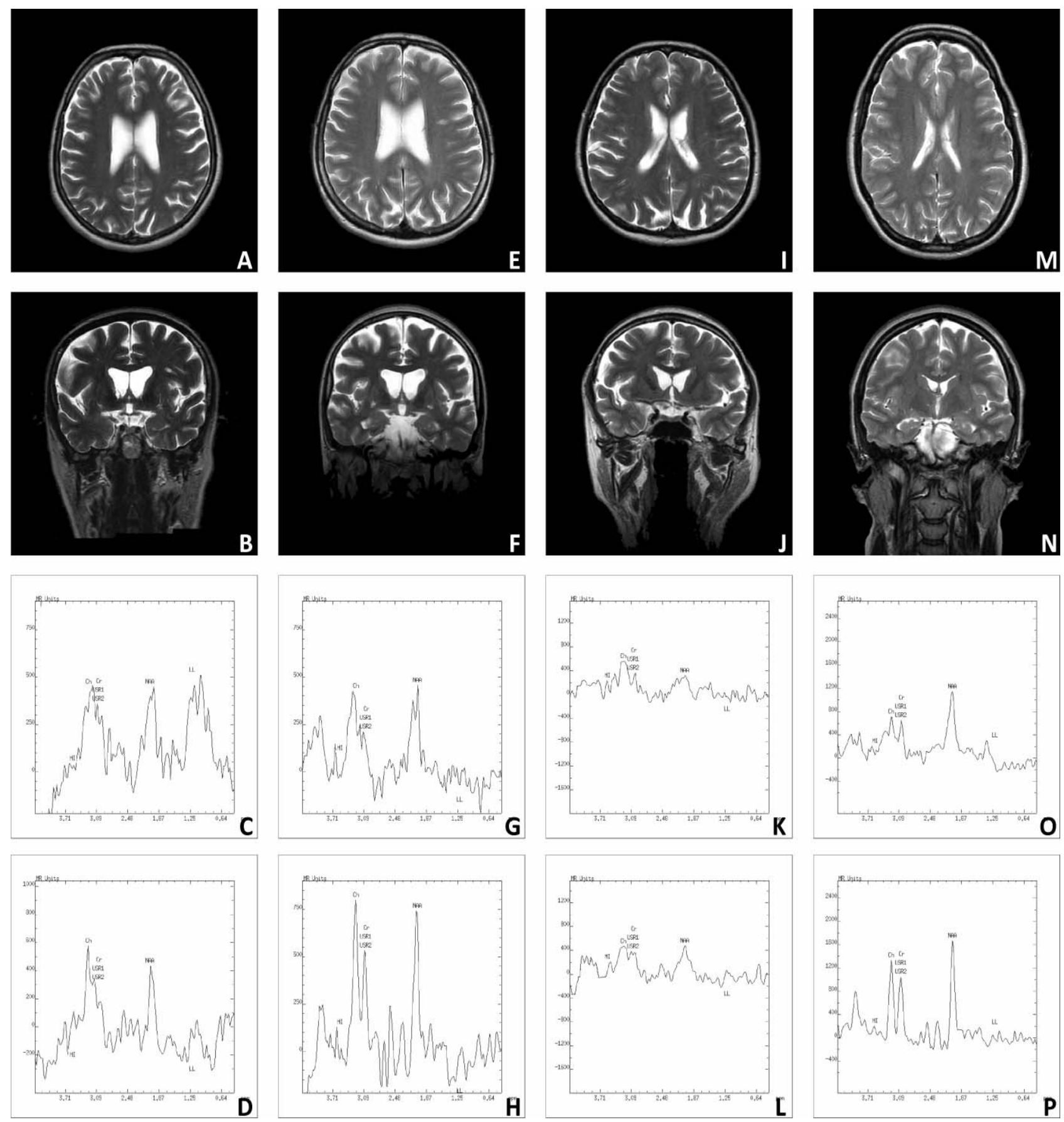

Axial and coronal MRI brain slices from the patient 1 before antibiotic therapy (A, B), after treatment (E, F), patient 2 (adultonset HD with the same number of high allele CAG triplets and the disease duration as the Patient 1) and patient 3 (19-year old-pre-HD woman with 40 high allele CAG triplets). A and B shows a bilateral multifocal signal hyperintensities (SH), more evident in the white matter of the left frontal lobe (A); and coronal T2 FSE section showing SH and cortical and subcortical atrophy (B). E and F shows mild resolution of SH after antibiotic treatment. A, B, E and F demonstrate striatal atrophy producing an increase in intercaudal distance and ventricular enlargement (,box-shape“) without putaminal hypointensities on T2. I and J (patient 2) show less prominent cortico-subcortical atrophy without SH. There is no SH in the white matter neither atrophy of the striatum on $\mathrm{M}$ and $\mathrm{N}$ (patient 3). Representative 1H-MRS spectra from the patient 1 before antibiotic therapy $(\mathrm{C}, \mathrm{D})$ and after antibiotic treatment $(\mathrm{G}, \mathrm{H})$, patient $2(\mathrm{~K}, \mathrm{~L})$ and patient $3(\mathrm{O}, \mathrm{P})$. Per our group findings, lactate/lipids (LL)/creatine ratio (LL/Cr) was displayed significantly higher in the patient 1 before antibiotic therapy as compared to patient 1 after treatment, patient 2 and patient 3.

Legend: LL - lactate/lipids; Cho - choline; Cr - creatine; mI - myo-inositol; NAA - N-acetylaspartate; pre-HD - premanifest Huntington's disease.

Figure 1. Brain magnetic resonance imaging (MRI) 
Table 1. Metabolite ratio in HD-LNB, adult-onset HD, pre-HD patients and normal subjects

\begin{tabular}{|c|c|c|c|c|c|c|}
\hline \multirow[b]{2}{*}{ Metabolite ratio } & & \multicolumn{2}{|c|}{ Patient 1} & \multirow{2}{*}{$\begin{array}{c}\text { Patient } 2 \\
\text { adult-onset HD }\end{array}$} & \multirow{2}{*}{$\begin{array}{c}\text { Patient } 3 \\
\text { pre-HD }\end{array}$} & \multirow{2}{*}{$\begin{array}{l}\text { Normal } \\
\text { metabolite } \\
\text { ratios }\end{array}$} \\
\hline & & $\begin{array}{c}\text { HD-LNB } \\
\text { pretreatment }\end{array}$ & $\begin{array}{c}\text { HD-LNB } \\
\text { postreatment }\end{array}$ & & & \\
\hline \multirow[t]{2}{*}{$\mathrm{NAA} / \mathrm{Cr}$} & L putamen & 1.50 & 1.66 & 1.35 & 2.23 & $1.13 \pm 0.06$ \\
\hline & FWM & 1.30 & 1.59 & 1.22 & 1.55 & $1.58 \pm 0.16$ \\
\hline \multirow[t]{2}{*}{ Cho/Cr } & Putamen & 1.24 & 2.81 & 2.28 & 1.37 & $0.73 \pm 0.07$ \\
\hline & FWM & 1.92 & 1.86 & 1.43 & 1.27 & $0.83 \pm 0.07$ \\
\hline \multirow[t]{2}{*}{$\mathrm{mI} / \mathrm{Cr}$} & Putamen & 0.10 & 0.25 & 0.43 & 0.35 & $0.48 \pm 0.04$ \\
\hline & FWM & 0.31 & 0.17 & 0.28 & 0.18 & $0.60 \pm 0.07$ \\
\hline \multirow[t]{2}{*}{$\mathrm{LL} / \mathrm{Cr}$} & Putamen & 3.30 & 1.59 & 0.80 & 1.34 & $0.62 \pm 0.41$ \\
\hline & FWM & 2.80 & 1.12 & 1.10 & 0.44 & $0.62 \pm 0.41$ \\
\hline
\end{tabular}

NAA - N-acetylaspartate; Cr - creatine; Cho - choline; $\mathrm{mI}$ - myo-inositol; $\quad$ LL - lactate/lipids; FWM - frontal white matter;

L - putamen left putamen; HD - Huntington's disease; LNB - Lyme neuroborreliosis

a sign characteristic of HD (Figure $1 F$ ) (Sturrock et al. 2010). A lactate diminished in the putamen and the deep white matter, but trace amount of lactate was noted in the putamen. A glutamine-glutamate (Glx) peak was detected and probably reflecting the release a Glx that are believed to occur during the acute-subacute period od neuroboreliosis. The foufold choline elevation are believed to occur during the subacute period of neuroborreliosis. A mI levels depleted in the white matter, and NAA/Cr ratio was normal (Figure 1G, H). The result of a genetic test for HD was positive (43 repeated high allele and 20 low allele CAG triplets) and the diagnosis of adult-onset HD was established.

\section{Patient 2}

A 53-year old woman with a positive family history for HD presented with chorea and mood disturbances. Brain MRI showed striatal atrophy (Figure 1I, J). The results of a genetic test for HD was positive (42 repeated high allele and 17 low allele CAG triplets). $1 \mathrm{H}-$ MRS showed slightly elevated resonances of choline and reduced $\mathrm{mI} / \mathrm{Cr}$ (Figure 1K, L).

\section{Patient 3}

A 19-year old woman with a family history of HD approached to take a presymptomatic testing for HD. After three separate, several hours long councelling sessions and preparation for testing, she has been evaluated neurologically, neuropsychologically and psychiatrically. Clinical examination and MRI evaluation were normal (Figure 1M, N). 1H-MRS showed nonsignificantly elevated $\mathrm{Cho} / \mathrm{Cr}$ and slightly reduced $\mathrm{mI} / \mathrm{Cr}$. Genetic test for HD was positive (40 repeated high allele and 15 low allele). Owing to genetic test, she was diagnosed as having pre-HD.

\section{DISCUSSION}

The ability of $1 \mathrm{H}-\mathrm{MRS}$ to track spectral changes makes it potentially useful for diagnostics and prognostics or as a tool to monitor the effects of treatment when evaluating new therapies, as well as additional tool when monitoring cognitive function in patients with HD (Sešok et al. 2014). The fact that $1 \mathrm{H}$-MRS has the ability in tracking biochemical changes after therapeutic interventions, offers the possibility of evaluation of the time course of the spectral changes and the efficacy of antibiotic treatment in patients with neuroborreliosis as well as cognitive function in HD after treatment. Extrapyramidal symptoms occurs in less than 2 percent of patients who manifested neurological complications associated to neuroborreliosis (Kohlepp et al. 1989, Biesiada et al. 1998, Piccolo et al. 1998, Markeljevic et al. 2011, Janavs et al. 1998). Too few cases of movement disorders such as chorea and dystonia were described during the course of neuroborreliosis (Piccolo et al. 1998). The unique triad of movement abnormalities (dystonia-parkinsonism and restlesness) represented the initial and only symptoms of neuroborreliosis in patient 1 , and these symptoms completely resolved after antibiotic therapy. Various infectious etiologies were reported in association with dystonia, choreoathetosis and ballismus (Markeljevic et al. 2011). Abnormalities on brain MRI are infrequent in the course of neuroborreliosis. A rare tumefactive white matter lesions related to microgliosis can be seen which are rarely reversal following antibiotic treatment (Piccolo et al. 1998, Markeljevic et al. 2011). We initially identified SH over the white matter and cortico-subcortical atrophy in the first patient, while the striatal atrophy was overlooked in the absence of the putaminal hyperintensities on T2-weighted images and increased diffusion coefitient in the striatum. These signs are most characteristic of hyperkinetic HD. Linear change in the caudate volume is systematically used as an early biomarker of HD pathology from many years before disease onset, and it may be used for selection of mutation carriers and for assesment of conversion of pre-HD to symptomatic HD (Hobbs et al. 2010). Nonspecific SH on initial MRI in the patient 1 , were consistent to probable ischemic lesions and gliosis consequentional to the development of vasculitis. Putaminal concentration of $\mathrm{mI}$ and NAA have been 
reported as potential biomarkers of HD onset and progression (Sturrock et al. 2010). The literature does not include any report correlating clinical and $1 \mathrm{H}-\mathrm{MRS}$ findings of patients with HD accompanied with neuroborreliosis. Spectral changes that have been seen in all $\mathrm{HD}$ individuals were restricted to the putamen and the white matter. A Lac peaks in spectra localised to the putamen and white matter of the patient 1 was sufficient to explain the presence of an acute or subacute metabolic changes (significant axonal loss and gliosis), with no evident neuronal loss. A Lac resonance probably increased following a hypoxic-ischemic insults, as a result of an anaerobic glycolysis, in which neurons developed energy failure associated to neuroborreliosis. Although the trace amounts of lactate was evident after antibiotic treatment, Lac diminished probably as a result of restored perfussion. A glutamine-glutamate peak that was detected after antibiotic treatment probably reflecting the release of glutamate that occurs in acute-subacute phase of neuroboreliosis. The fourfold Cho elevation after treatment are believed to occur during the subacute period of neuroborreliosis and might be interpreted to be associated with axonal injury and gliosis.

To our knowledge, no proton spectra similar to those presented in this patient have been reported to represent brain changes in HD patients accompanied with neuroborreliosis. Therefore, it helps to be aware of an therapeutic effects in neuroborreliosis when interpreting $1 \mathrm{H}$ MRS findings. Some authors (Ustymovicz et al. 2004) found a significant increase in $\mathrm{Cho/Cr}$ and mild increase in $\mathrm{mI}$ in patients with neuroborreliosis. Their $\mathrm{LL} / / \mathrm{Cr}$ and NN/Cr were similar to those of the control group, except for those patient who had abnormal MRI findings. Recently, putaminal mI (osmolyte and glial marker) was identified as an biomarker of early HD (Sturrock et al. 2010). These data may be extended to pre-HD subjects, but are insufficient to be correlated with number of CAG triplets and symptoms duration. In all presented HD subjects, mI levels were slightly decreased, probably as a result of hypoosmolar states and chronic hypoxic encephalopathy. Vasculitis was reported as the main pathogenetic mechanism of basal ganglia ischaemia related to neuroborreliosis. Other proposed mechanisms include vasculopathy due to direct basal ganglia invasion by the organism, neuronal injury by an cytotoxin, antibiodies cross-reactivity with basal ganglia epitopes (Janavs et al. 1998). The selective pathophysiology that occurs in HD is still unknown. Literature report suggested a role of metabolic dysfunction and oxidative damage which are dominantly restricted to the basal ganglia in the pathogenesis of $\mathrm{HD}$, while other brain regions are relatively unaltered (Walker et al. 2007). Oxidative damage of basal ganglia in HD may make them more susceptible for further oxidative stress caused by infection, acting as a trigger of movement disorders associated to neuroborreliosis. Antibiotic therapy temporarily improved movement abnormalities; eliminated the serum and CSF antibodies; and delayed underlying diagnosis of $\mathrm{HD}$.
These findings provide further evidence of the global energy metabolism failure, diffuse axonal loss, proliferation of activated microglia and astrogliosis in HD individuals (Piccolo et al. 1998, Harms et al. 1997, Jenkins et al. 1998, Reynolds et al. 2005).

\section{CONCLUSION}

Decreased $\mathrm{mI}$ and elevated Cho in HD subjects on MRS suggest global cerebral energy failure, diffuse axonal injury and gliosis. In patients with $\mathrm{HD}$ and neuroborreliosis, abnormal spectroscopic findings before antibiotic treatment include Lac elevation, glutamine-glutamate elevation, and reduced $\mathrm{mI}$. The findigs of the lactate peak in patient with HD and LNB before antibiotic tratment and choline elevation after treatment confirm the specific 1H-MRS spectral pattern of neuroborreliosis associated with MRI abnormalities reported previously. Dramatic spectral improvement observed under antibiotics show that the spectral specificity is valid only for untreated patients. In line with this findings, we suggest that treatment in HD should be directed not only against disturbed energy metabolism, but also towards axonal protection.

\section{Acknowledgements:}

This work has been fully supported by the Croatian Science Foundation under the project number IP-092014-2979

\section{Conflict of interest:}

Research funding was provided by Croatian Science Foundation (project code: MODERN, \# 2979).

Financial disclosures: Neven Henigsberg has participated in clinical trials sponsored by Lundbeck, Takeda, GlaxoSmithKline, and Pfizer and holds research grants from Croatian Science Foundation and University of Zagreb.

Patient consent form been signed by the patient.

\section{Contribution of individual authors:}

Helena Sarac: had full access to all of the data in the study and takes responsibillity for the integrity of the data and the accuracy of the data analysis;

Helena Sarac \& Neven Henigsberg: study concept and design;

Helena Sarac, David Ozretic, Neven Henigsberg, Pero Hrabac, Ivan Bagaric \& Lucija Bagaric-Krakan: acquisition, analysis, or interpretation of data;

Helena Sarac \& Neven Henigsberg: drafting of the manuscript;

Helena Sarac, David Ozretic, Neven Henigsberg, Pero Hrabac, Ivan Bagaric \& Lucija BagaricKrakan: critical revision of the manuscript for important intellectual content;

Helena Sarac, David Ozretic, Neven Henigsberg, Pero Hrabac, Ivan Bagaric \& Lucija BagaricKrakan: study supervision. 


\section{References}

1. Sturrock A, Laule C, Decolongon J, Dar Santos $R$, Coleman AJ, Creighton S, Bechtel N, Reilmann R, Hayden MR, Tabrizi SJ, Mackay AL, Leavitt BR: Magnetic resonance spectroscopy biomarkers in premanifest and early Huntington disease. Neurology 2010; 75:1702-10.

2. Ustymowicz A, Tarasów E, Zajkowska J, Walecki J, Hermanowska-Szpakowicz T: Proton MR spectroscopy in neuroborreliosis: a preliminary study. Neuroradiology 2004; 46:26-30.

3. Sešok S, Bolle N, Kobal J, Bucik V, Vodušek DB: Cognitive function in early clinical phase Huntington disease after rivastigmine treatment. Psychiatr Danub 2014; 26:239-48.

4. Kohlhepp W, Kuhn W, Krüger H: Extrapyramidal features in central Lyme borreliosis. Eur Neurol 1989; 29:150-5.

5. Biesiada G, Czapiel J, Sobczyk-Krupiarz I, Garlicki A, Mach T: Neuroborreliosis with extrapyramidal symptoms: a case report. Pol Arch Med Wewn 1998; 118:314-7.

6. Piccolo I, Thiella G, Sterzi R, Colombo N, Defanti CA. Chorea as a symptom of neuroborreliosis: a case study. Ital J Neurol Sci 1998; 19:235-9.

7. Markeljevic J, Sarac H, Rados M: Tremor, seizures and psychosis as presenting symptoms in a patient with chronic lyme neuroborreliosis (LNB). Coll Antropol 2011; 35(Suppl 1):313-8.

8. Janavs JL, Aminoff MJ: Dystonia and chorea in acquired systemic disorders. J Neurol 13 Neurosurg Psychiatry 1998; 65:436-445.

9. Hobbs NZ, Barnes J, Frost C, Henley SM, Wild EJ, Macdonald K, Barker RA, Scahill RI, Fox NC, Tabrizi SJ: Onset and progression of pathologic atrophy in Huntington disease: a longitudinal MR imaging study. AJNR Am J Neuroradiol 2010; 31:1036-41.

10. Walker FO: "Huntington's disease". Lancet 2007; 369:218-28.

11. Harms L, Meierkord H, Timm G, Pfeiffer L, Ludolph AC: Decreased $\mathrm{N}$-acetyl-aspartate/choline ratio and increased lactate in the frontal lobe of patients with Huntington's disease: a proton magnetic resonance spectroscopy study. J Neurol Neurosurg Psychiatry 1997; 62:27-30.

12. Jenkins BG, Rosas HD, Chen YC, Makabe T, Myers R, MacDonald M, Rosen BR, Beal MF, Koroshetz WJ: $1 H$ NMR spectroscopy studies of Huntington's disease: correlations with CAG repeat numbers. Neurology 1998; 50:1357-65.

13. Reynolds NC Jr, Prost RW, Mark LP: Heterogeneity in 1 HMRS profiles of presymptomatic and early manifest Huntington's disease. Brain Res 2005; 1031:82-9.

\section{Correspondence:}

Helena Sarac, MD, PhD

Department of Neurology, University Hospital Zagreb

HR-Kispaticeva 12, 10000 Zagreb, Croatia

E-mail:helenasarac57@gmail.com 